\title{
GMR
}

\section{Copy number variations in spermatogenic failure patients with chromosomal abnormalities and unexplained azoospermia}

\author{
Y. Dong ${ }^{1,2}$, Y. Pan' ${ }^{1}$, R. Wang ${ }^{1}$, Z. Zhang ${ }^{1}$, Q. Xi' ${ }^{1}$ and R.-Z. Liu' ${ }^{1}$ \\ ${ }^{1}$ Center for Reproductive Medicine, Center for Prenatal Diagnosis, First Hospital, \\ Jilin University, Changchun, China \\ 2Department of Genetics and Metabolism, Beijing Obstetrics and Gynecology Hospital, \\ Capital Medical University, Beijing Maternal and Child Health Care Hospital, \\ Beijing, China \\ Corresponding author: R.-Z. Liu \\ E-mail: Irz410@126.com
}

Genet. Mol. Res. 14 (4): 16041-16049 (2015)

Received June 16, 2015

Accepted September 29, 2015

Published December 7, 2015

DOI http://dx.doi.org/10.4238/2015.December.7.17

ABSTRACT. Male infertility is mostly caused by spermatogenic failure. Currently, routine genetic analyses of unexplained azoospermia or oligozoospermia are limited to the investigation of $Y$ chromosomal microdeletions and chromosome karyotype analyses. The aim of this study was to find spermatogenic failure genes in patients with chromosomal abnormalities and unexplained azoospermia caused by copy number variations in order to provide a theoretical basis for further research. Spermatogenic failure patients consisting of 13 males with chromosomal abnormalities and 20 with unexplained azoospermia were enrolled. The subjects underwent high-throughput genome-wide sequencing to find copy number variants (CNVs), and the results were analyzed using the Database of Genomic Variants, Online Mendelian Inheritance in Man database, and PubMed. The results showed that $16 \mathrm{CNVs}$ were detected in 11 patients with chromosome abnormalities, and $26 \mathrm{CNVs}$ were found in 16 males with azoospermia. Our data showed CNV-involved loci including: three times on $11 p 11.12$ and 14q11.2 and twice on 6p21.32, 13q11, 15q11.11, 16p12.2, 
and 21q22.3. Some CNVs may involve changes in genetic structure and function or gene mutations, which may affect gene expression in testicular tissues and lead to spermatogenic failure. The involved genes include EDDM3A, EDDM3B, HLA-DRB1, HLA-DQA1, POTE B, GOLGA8C, DNMT3L, ALF, NPHP1, NRG1, RID2, ADAMTS20, TWF1, COX10, MAK, and DNEL1. By applying high throughput genome-wide sequencing to determine CNVs, we provide a number of candidate genes possibly contributing to spermatogenic failure.

Key words: Copy number variations; Spermatogenic failure; Chromosomal abnormalities; Y-chromosomal microdeletions; Azoospermia; Oligozoospermia

\section{INTRODUCTION}

Male infertility, which refers to a male's inability to cause pregnancy in a fertile female, is mostly caused by spermatogenic failure. Problems during spermatogenesis contribute to a lower or absent production of spermatozoa, are determined by routine semen analysis, and described using terms such as 'azoospermia', 'oligozoospermia', 'teratozoospermia', or 'asthenozoospermia', or a combination of the above ('oligoasthenozoospermia', 'oligoteratozoospermia', or 'oligoasthenoteratozoospermia') (Massart et al., 2012).

Currently, clinical diagnosis of unexplained azoospermia or oligozoospermia involving a routine genetic analysis is limited to the investigation of $Y$ chromosomal microdeletions and chromosome karyotype analyses. The incidence of $\mathrm{Y}$ chromosomal microdeletions and chromosome abnormalities is $\sim 7.4$ and $\sim 5 \%$ in male infertility patients, and the prevalence is increased to $\sim 9.7$ and $\sim 13 \%$ in azoospermic males (Van Assche et al., 1996, Ferlin et al., 2007; McLachlan and O'Bryan, 2010).

Recently, gene mutations and polymorphisms, unbalanced translocations, and protamine defects relevant to spermatogenesis have been elucidated (Carrell et al., 2006). The current study includes two groups of infertile patients without $Y$ chromosomal microdeletions, with one group only having chromosome abnormalities. Martin et al. (2008) suggested a possible role of additional copy number variants (CNVs) in chromosome inversions and translocation events. Therefore, we tried to determine whether there is any other cause of spermatogenic failure other than abnormal karyotype. The other group of patients has unexplained azoospermia, and we also attempt to explain the possible pathogenesis of problems during spermatogenesis.

CNV is defined as a DNA segment longer than $1 \mathrm{~kb}$ with a variable copy number compared with a reference genome (Feuk et al., 2006; Freeman et al., 2006). The research methods for CNVs include fluorescent in situ hybridization (FISH), comparative genomic hybridization (CGH), multiplex amplifiable probe hybridization (MAPH), multiplex ligation-dependent probe amplification (MLPA), etc. CNVs have recently been shown to be an important source of genetic diversity, exhibiting remarkable differences between individuals and playing a role in complex diseases such as mental retardation, schizophrenia, and cancer (Redon et al., 2006; Lee et al., 2007; Fanciulli et al., 2010).

Recently, CNVs have also been applied to the analysis of male infertility by CGH (Lee et al., 2007; Tuttelmann et al., 2011). However, to the best of our knowledge, little information is available regarding CNVs in males with chromosomal abnormalities and unexplained azoospermia using high throughout genome-wide sequencing. Hence, we will focus on those cases in order to determine the pathogenesis of spermatogenic failure. 


\section{MATERIAL AND METHODS}

\section{Patients}

Spermatogenic failure patients with chromosomal abnormalities $(\mathrm{N}=13)$ and unexplained azoospermia $(\mathrm{N}=20)$ were enrolled. The mean ages were 29.0 years (23-37 years) and 27.6 years (22-39 years). All men with known clinical problems (e.g., undescended testes, varicocele, retrograde ejaculation, infections, and obstructive azoospermia), abnormal examination results (reproductive hormone levels, serum inhibin-B, seminal plasma fructose, and $\alpha$-glycosidase levels), and genetic causes of spermatogenic failure (chromosome karyotype anomalies, Y-chromosomal mircodeletions) were excluded. All participants gave written informed consent for evaluation of their clinical data and genetic analyses of their donated DNA samples according to a protocol approved by the Chinese Association of Humanitarianism and Ethics.

\section{Semen analysis}

Semen samples were obtained after a 3-7 day period of ejaculatory abstinence, and semen analyses were performed three times within an interval of three months according to the World Health Organization guidelines (5th) (World Health Organization, 2010). Absence of spermatozoa in the semen ejaculate, if detected three times, was considered azoospermia (A). A sperm concentration of $<15 \times 10^{6} / \mathrm{mL}$ was considered oligozoospermia (O). A sperm concentration of $<15 \times 10^{6} / \mathrm{mL}$ with progressive motility $<32 \%$ or total (progressive + nonprogressive) motility $<40 \%$ was considered oligoasthenozoospermia (OA). Oligoteratozoospermia (OT) was defined as a sperm concentration of $<15$ $x 10^{6} / \mathrm{mL}$ with a percentage of morphologically normal sperm of $<4 \%$. Oligoasthenoteratozoospermia (OAT) was defined as a sperm concentration of $<15 \times 10 \% \mathrm{~mL}$ progressive motility $<32 \%$ or total (progressive + nonprogressive) motility $<40 \%$, and a percentage of morphologically normal sperm of $<4 \%$.

\section{High throughput genome-wide sequencing and data analysis}

Peripheral blood $(1 \mathrm{~mL})$ containing EDTA $(2.25 \mathrm{mg} / \mathrm{mL}$ ethylenediamine tetra-acetic acid) was sent to Berry Genomics, Beijing, China for processing and high throughput sequencing to determine the CNVs. The results were analyzed using the Database of Genomic Variants (DGV) (Genomic Variants in Human Genome Build GRCh37: Feb. 2009, hg19) (http://dgv.tcag.ca/dgv/ app/home), OMIM (Online Mendelian Inheritance in Man) (http://omim.org/), and PubMed (Key words were infertility, spermiogenesis, azoospermia, oligozooospermia, and testis).

\section{RESULTS}

In the present study, $16 \mathrm{CNVs}$ were detected in 11 patients with chromosome abnormalities, and $26 \mathrm{CNVs}$ were found in 16 males with azoospermia. There were no obvious abnormalities in the remaining two chromosomal abnormality patients (Nos. 8136 and 8548) (Table 1) and the other four unexplained azoospermic males (Nos. 7742, 7958, 8063, and 8077) (Table 2). CNV sizes ranged from $100 \mathrm{~kb}$ to $2.38 \mathrm{Mb}$ and $40 \mathrm{~kb}$ to $2.2 \mathrm{Mb}$ in patients with chromosome abnormalities and azoospermia, respectively. For chromosome abnormality carriers, as shown in Table 1, out of 13 patients, five cases were diagnosed with azoospermia (A), three with oligoasthenozoospermia 
(OA), two with oligozoospermia (O), two with oligoasthenoteratozoospermia (OAT), and one with oligoteratozoospermia (OT).

Our data showed CNV-involved loci three times on $11 \mathrm{p} 11.12$ and $14 \mathrm{q} 11.2$, twice on $6 \mathrm{p} 21.32$, $13 q 11,15 q 11.1-11.2,16 p 12.2,21 q 22.3$, and once on 1p33, 1p36.21, 2p16.3, 2q13, 2q21.1, 2q36.1, 3p14.1, 4q13.2, 5q11.1, 5q23.1, 5q35.3, 6q22.31, 7p21.3, 7q31.1, 8p12, 8p22, 9p21.1, 10p11.1, $10 q 11.22,10 q 21.1,12 q 12,12 q 14.1,14 q 24.3,15 q 25.1,17 p 12$, and 22q11.21 (Figure 1).

\begin{tabular}{|c|c|c|c|c|c|c|}
\hline Case No. & Karyotypes by G-banding & Semen analysis & Sequencing results & Type & Size (bp) & Regions on chromosomes \\
\hline 7050 & $46, \mathrm{XY}, \mathrm{t}(1 ; 2)(\mathrm{q} 21 ; \mathrm{p} 23)$ & $\mathrm{OA}$ & chr16:(21960001-22440000)x1 & Loss & $480 \mathrm{~K}$ & $16 \mathrm{p} 12.2$ \\
\hline 8004 & $46, X Y, t(2 ; 8)(q 31 ; q 22)$ & OAT & chr2:(110860001-111000000)x1 & Loss & $140 \mathrm{~K}$ & $2 q 13$ \\
\hline 5107 & $45, \mathrm{XY},-13,-19,+\operatorname{der}(19) \mathrm{t}(13 ; 19)(\mathrm{q} 12 ; \mathrm{p} 13)$ & A & chr6:(121500001-121600000)x1 & Loss & $100 \mathrm{~K}$ & $6 q 22.31$ \\
\hline \multirow[t]{2}{*}{5118} & $46, X Y, t(X ; 2)(p 22 ; p 11)$ & A & chr2:(52100001-52280000)x1 & Gain & $180 \mathrm{~K}$ & $2 \mathrm{p} 16.3$ \\
\hline & & & chr6:(32460001-32580000)×3 & Gain & $120 \mathrm{~K}$ & $6 \mathrm{p} 21.32$ \\
\hline 6632 & $45, X, \operatorname{der}(Y ; 22)(q 10 ; q 10)$ & $\mathrm{OA}$ & chr9:(29520001-29960000)x3 & Gain & $440 \mathrm{~K}$ & $9 p 21.1$ \\
\hline 7504 & $46, \mathrm{XY}, 21 \mathrm{pstk}-$ & OT & chr22:(21100001-21460000)x1 & Loss & $360 \mathrm{~K}$ & $22 q 11.21$ \\
\hline 8557 & $46, X Y, 21 p-$ & $A$ & chr21:(46740001-48100000)x1 & Loss & $1.36 \mathrm{M}$ & $21 \mathrm{q} 22.3$ \\
\hline \multirow[t]{2}{*}{8033} & $46, X Y, 16 q h+$ & OAT & chr14:(73940001-74140000)x1 & Loss & $200 K$ & $14 q 24.3$ \\
\hline & & & chr15:(79000001-79100000)x1 & Loss & $100 \mathrm{~K}$ & $15 q 25.1$ \\
\hline 7523 & $46, X Y, \operatorname{inv}(1)(p 32 q 42)$ & $A$ & chr17:(13760001-14200000)x3 & Gain & $440 \mathrm{~K}$ & $17 \mathrm{p} 12$ \\
\hline \multirow[t]{3}{*}{4894} & $46, X Y, \operatorname{inv}(1)(p 36 q 25), \operatorname{inv}(9)(p 11 q 12)$ & $A$ & chr2:(132340001-133020000)x1 & Loss & $680 \mathrm{~K}$ & $2 \mathrm{q} 21.1$ \\
\hline & & & chr11:(48820001-49400000)x3 & Gain & $580 \mathrm{~K}$ & $11 \mathrm{p} 11.12$ \\
\hline & & & chr13:(19440001-19640000)x3 & Gain & $200 \mathrm{~K}$ & $13 q 11$ \\
\hline \multirow[t]{2}{*}{3849} & $47, \mathrm{XY},+\mathrm{mar}$ & 0 & chr10:(38480001-39160000)×3 & Gain & $680 \mathrm{~K}$ & 10p11.1 \\
\hline & & & chr15:(20180001-22560000)x3 & Gain & $2.38 \mathrm{M}$ & $15 q 11.1-q 11.2$ \\
\hline 8136 & $46, X Y, t(1 ; 3)(p 22 ; q 29)$ & $\mathrm{OA}$ & No obvious abnormalities & & & \\
\hline 8548 & $46, X Y, 13 p+$ & 0 & No obvious abnormalities & & & \\
\hline
\end{tabular}

$\mathrm{A}=$ azoospermia; $\mathrm{O}=$ oligozoospermia; $\mathrm{OA}=$ oligoasthenospermia; OT = oligoteratozoospermia; OAT = oligoastenoteratozoospermia.

Table 2. Detailed analysis of 20 patients with unexplained azoospermia.

\begin{tabular}{|c|c|c|c|c|}
\hline Case No. & Sequencing results & Type & Size(bp) & Regions on chromosomes \\
\hline \multirow[t]{2}{*}{7516} & chr8:(31500001-32040000)x3 & Gain & $540 \mathrm{~K}$ & $8 p 12$ \\
\hline & chr12:(38580001-38740000)x1 & Loss & $160 \mathrm{~K}$ & $12 q 12$ \\
\hline 7524 & chr1: (15300001-15480000)×3 & Gain & $180 \mathrm{~K}$ & 1p36.21 \\
\hline 7647 & chr5: (49440001-49480000)x1 & Loss & $80 \mathrm{~K}$ & $5 q 11.1$ \\
\hline \multirow[t]{2}{*}{7688} & chr7: (12860001-13100000)x1 & Loss & $240 \mathrm{~K}$ & $7 \mathrm{p} 21.3$ \\
\hline & chr11: (49240001-49980000)x1 & Loss & $740 \mathrm{~K}$ & $11 \mathrm{p} 11.12$ \\
\hline 7937 & chr2: (222200001-223020000)×3 & Gain & $820 \mathrm{~K}$ & $2 q 36.1$ \\
\hline 7998 & chr4: (70180001-70440000)×3 & Gain & $260 \mathrm{~K}$ & $4 q 13.2$ \\
\hline 8064 & chr7: (112180001-112360000)x1 & Loss & $180 \mathrm{~K}$ & $7 q 31.1$ \\
\hline \multirow[t]{2}{*}{8080} & chr13: (19440001-19500000)x1 & Loss & $60 \mathrm{~K}$ & $13 q 11$ \\
\hline & chr16: (22400001-22720000)x1 & Loss & $320 \mathrm{~K}$ & 16p12.2 \\
\hline \multirow[t]{2}{*}{8196} & chr5: (117380001-117660000)x1 & Loss & $280 \mathrm{~K}$ & $5 q 23.1$ \\
\hline & chr12: (60680001-60940000)x1 & Loss & $260 \mathrm{~K}$ & 12q14.1 \\
\hline \multirow[t]{3}{*}{8235} & chr10: $(46980001-47700000) \times 3$ & Gain & $720 \mathrm{~K}$ & $10 q 11.22$ \\
\hline & chr10: (59560001-59760000)×1 & Loss & $200 K$ & 10q21.1 \\
\hline & chr21: (44700001-45240000)×3 & Gain & $540 \mathrm{~K}$ & $21 q 22.3$ \\
\hline \multirow[t]{2}{*}{7467} & chr1: (47580001-47680000)x1 & Loss & $100 \mathrm{~K}$ & $1 p 33$ \\
\hline & chr8: (13720001-14040000)x3 & Gain & $320 \mathrm{~K}$ & 8p22 \\
\hline \multirow[t]{2}{*}{7468} & chr5: (180400001-180440000)x1 & Loss & $40 \mathrm{~K}$ & $5 q 35.3$ \\
\hline & chr14: (19460001-20420000)x3 & Gain & $960 \mathrm{~K}$ & $14 q 11.2$ \\
\hline 7477 & chr14: (19460001-20280000)x3 & Gain & $820 \mathrm{~K}$ & $14 q 11.2$ \\
\hline \multirow[t]{2}{*}{7333} & chr3: (65780001-66340000)×3 & Gain & $560 \mathrm{~K}$ & 3p14.1 \\
\hline & chr14: (19460001-20440000)×3 & Gain & $980 \mathrm{~K}$ & $14 q 11.2$ \\
\hline \multirow[t]{2}{*}{6035} & chr6: (32440001-32700000)×3 & Gain & $260 \mathrm{~K}$ & $6 p 21.32$ \\
\hline & chr11: (48800001-49380000)×3 & Gain & $580 \mathrm{~K}$ & $11 \mathrm{p} 11.12$ \\
\hline 5190 & chr15: (20180001-22380000)x3 & Gain & $2.2 \mathrm{M}$ & $15 q 11.1$ \\
\hline 7742 & No obvious abnormalities & & & \\
\hline 7958 & No obvious abnormalities & & & \\
\hline 8063 & No obvious abnormalities & & & \\
\hline 8077 & No obvious abnormalities & & & \\
\hline
\end{tabular}




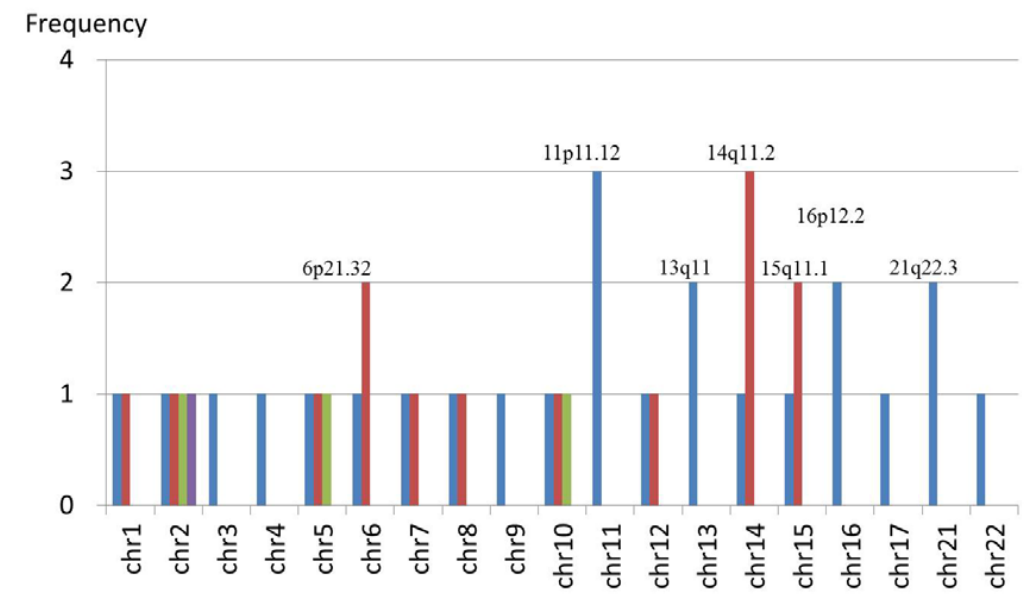

Figure 1. Frequency of CNVs on different regions of chromosomes. CNVs occurred on $11 p 11.12$ and $14 q 11.2$ three times; on 6p21.32, 13q11, 15q11.1, 16p12.2, and 21q22.3 twice; and on 1p33, 1p36.21, 2p16.3, 2q13, 2q21.1, 2q36.1, 3p14.1, 4q13.2, 5q11.1, 5q23.1, 5q35.3, 6q22.31, 7p21.3, 7q31.1, 8p12, 8p22, 9p21.1, 10p11.1, 10q11.22, 10q21.1, $12 q 12,12 q 14.1,14 q 24.3,15 q 25.1,17 p 12$, and 22q11.21 only once.

\section{DISCUSSION}

In this present study, $42 \mathrm{CNVs}$ were detected in 33 cases of infertile males by high throughput genome-wide sequencing. Among them, there were $16 \mathrm{CNVs}$ in 13 males with chromosomal abnormalities and $26 \mathrm{CNVs}$ in 20 unexplained azoospermic patients. Therefore, it is important to differentiate between CNVs that are likely to contribute to an affected individual's spermatogenic failure and CNVs that may be polymorphism variations. Then, the loci and lociassociated genes of CNVs were examined using DGV, OMIM database, and PubMed with the following key words: infertility, azoospermia, oligozoospermia, spermiogenesis, and testis. The loci related to the spermatogenic failure gene will be analyzed emphatically.

CNVs on the regions of $11 p 11.12,13 q 11,16 p 12.2,1 p 33,1 p 36.21,2 q 21.1,2 q 36.1$, 3p14.1, 4q13.2, 5q11.1, 5q23.1, 5q35.3, 7p21.3, 7q31.1, 8p22, 9p21.1, 10p11.1, 10q11.22, $10 q 21.1,12 q 14.1,14 q 24.3,15 q 25.1$, and 22 q11.21 on spermatogenic failure-related genes were not discovered by the time of this literature reported. However, CNVs associated with the genes of spermatogenesis impairment on 14q11.2, 6p21.32, 15q11.2, 21q22.3, 2p16.3, 2q13, 6q22.31, 8p12, 12q12, and 17p12 are shown in Table 3.

The CNVs in three patients, Nos. 7468,7477 , and 7333, on $14 q 11.2$ involved EDDM3A and EDDM3B. Damyanova et al. (2013) reported that the loss of chr.14q11.2 can affect the proteins that are synthesized and secreted by epididymal epithelial cells that have been found to be up-regulated in the epididimis of non-obstructive azoospermia (NOA) men. In spite of the gains exhibited in our three patients, the CNVs may also affect the function of the genes and lead to azoospermia.

Patient Nos. 6035 and 5118 exhibited CNVs on 6p21.32. Hu et al. (2014) investigated 3608 NOA cases and 5909 controls to identify additional risk loci, and found that $6 \mathrm{p} 21.32$ as one of the strong evidence of the NOA susceptibility loci. The CNVs on 6p21.32 involved OMIM genes, HLA-DRB1 and HLA-DQA1, which belong to human leukocyte antigen (HLA) class II genes. van 
der Ven et al. (2000) reported that patients with male factor infertility differ in their HLA class II allele constitution from males with normozoospermia. It was suggested that genes identical to or located in close vicinity to $H L A$ class II genes may influence spermatogenesis and male gamete function (van der Ven et al., 2000). Other studies have also reported that specific alleles and single nucleotide polymorphisms (SNPs) in the HLA region were associated with NOA. It is likely that variations in this $H L A$ class II region might mediate the response to testicular microenvironmental antigens and cause testicular azoospermia through autoimmune inflammatory responses (Kurpisz et al., 2011; Zhao et al., 2012; Jinam et al., 2013). This may be an explanation for the azoospermia observed in two of the patients.

\begin{tabular}{|c|c|c|c|c|c|c|}
\hline Case No. & Phenotype & Region & Type & Size (bp) & Polymorphisms from $\mathrm{DGV}^{*}$ & Gene symbols \\
\hline 768860354894 & $3 \times A$ & $11 \mathrm{p} 11.12$ & 1xloss/2xgain & $740 \mathrm{~K} / 580 \mathrm{~K} / 580 \mathrm{~K}$ & $Y$ & \\
\hline 746874777333 & $3 \times A$ & $14 q 11.2$ & 3xgain & $960 \mathrm{~K} / 820 \mathrm{~K} / 980 \mathrm{~K}$ & Y & $E D D M 3 A, E D D M 3 B[14]$ \\
\hline 60355118 & $2 \times A$ & $6 \mathrm{p} 21.32$ & $2 x$ gain & $260 \mathrm{~Kb} / 120 \mathrm{~K}$ & & $H L A-D R B 1, H L A-D Q A 1[16-19]$ \\
\hline 48948080 & $2 \times A$ & $13 q 11$ & 1xgain/1xloss & $200 \mathrm{~K} / 60 \mathrm{~K}$ & & \\
\hline 51903849 & $1 \times A / 1 \times O$ & $15 q 11.1-11.2$ & 2xgain & $2.2 \mathrm{M} / 2.38 \mathrm{M}$ & $\mathrm{Y}$ & POTE B[20-21], GOLGA8C[22] \\
\hline 80807050 & $1 \mathrm{xA} / 1 \mathrm{xOA}$ & $16 \mathrm{p} 12.2$ & 2xloss & $320 \mathrm{~K} / 480 \mathrm{~K}$ & Y & \\
\hline 82358557 & $2 \times A$ & $21 q 22.3$ & 1xgain/1xloss & $540 \mathrm{~K} / 1.36 \mathrm{M}$ & & DNMT3L[23] \\
\hline 7467 & $A$ & $1 \mathrm{p} 33$ & Loss & $100 \mathrm{~K}$ & & \\
\hline 7524 & $A$ & $1 \mathrm{p} 36.21$ & Gain & $180 \mathrm{~K}$ & & \\
\hline 5118 & $A$ & $2 p 16.3$ & Gain & $180 \mathrm{~K}$ & & $A L F[24]$ \\
\hline 8004 & OAT & $2 q 13$ & Loss & $140 \mathrm{~K}$ & & NPHP1[25-26] \\
\hline 4894 & $A$ & $2 \mathrm{q} 21.1$ & Loss & $680 \mathrm{~K}$ & & \\
\hline 7937 & $A$ & $2 q 36.1$ & Gain & $820 \mathrm{~K}$ & & \\
\hline 7333 & $A$ & 3p14.1 & Gain & $560 \mathrm{~K}$ & Y & \\
\hline 7998 & $A$ & $4 q 13.2$ & Gain & $260 \mathrm{~K}$ & & \\
\hline 7647 & $A$ & $5 q 11.1$ & Loss & $80 \mathrm{~K}$ & & \\
\hline 8196 & $A$ & $5 q 23.1$ & Loss & $280 \mathrm{~K}$ & & \\
\hline 7468 & A & $5 q 35.3$ & Loss & $40 \mathrm{~K}$ & $\mathrm{Y}$ & \\
\hline 5107 & $A$ & $6 q 22.31$ & Loss & $100 \mathrm{~K}$ & & \\
\hline 7688 & $A$ & $7 \mathrm{p} 21.3$ & Loss & $240 \mathrm{~K}$ & & \\
\hline 8064 & $A$ & $7 q 31.1$ & Loss & $180 \mathrm{~K}$ & Y & \\
\hline 7516 & $A$ & $8 \mathrm{p} 12$ & Gain & $540 \mathrm{~K}$ & & $N R G 1[27-28]$ \\
\hline 7467 & A & $8 p 22$ & Gain & $320 \mathrm{~K}$ & & \\
\hline 6632 & OA & $9 \mathrm{p} 21.1$ & Gain & $440 \mathrm{~K}$ & Y & \\
\hline 3849 & $A$ & $10 p 11.1$ & Gain & $680 \mathrm{~K}$ & & \\
\hline 8235 & $A$ & $10 q 11.22$ & Gain & $720 \mathrm{~K}$ & Y & \\
\hline 8235 & $A$ & $10 q 21.1$ & Loss & $200 K$ & Y & \\
\hline 7516 & $A$ & $12 q 12$ & Loss & $160 \mathrm{~K}$ & Y & RID2, ADAMTS20, TWF1[29] \\
\hline 8196 & $A$ & $12 q 14.1$ & Loss & $260 K$ & Y & \\
\hline 8033 & OAT & $14 q 24.3$ & Loss & $200 \mathrm{~K}$ & & \\
\hline 8033 & OAT & $15 q 25.1$ & Loss & $100 \mathrm{~K}$ & & \\
\hline 7523 & $A$ & $17 \mathrm{p} 12$ & Gain & $440 \mathrm{~K}$ & & COX10[30-31], MAK[32], DNEL1[33] \\
\hline 7504 & OT & $22 q 11.21$ & Loss & $360 \mathrm{~K}$ & Y & \\
\hline
\end{tabular}

$\mathrm{A}=$ azoospermia; $\mathrm{O}=$ oligozoospermia; $\mathrm{OA}=$ oligoasthenospermia; OT = oligoteratozoospermia; OAT = oligoastenoteratozoospermia. *In the DGV database, there were noquery results for diseases of CNVs (polymorphisms) showing whole red or blue lines. This suggests that the CNVs (including gains and losses) are also found in the normal population and are likely not pathogenic.

Guediche et al. (2012) detected small supernumerary marker chromosomes in patients using array $\mathrm{CGH}$ to evaluate the size and the precise genomic constitution. POTE B (POTE ankyrin domain family (member $B)$ ), located on $15 q 11.2$, is expressed in human testis, particularly in a specific cell type in primary spermatocytes (Ise et al., 2008). It is possible that POTE plays a role in human sperm maturation and its involvement in diverse regulatory pathways serves an important 
signaling function in the reproductive system (Guediche et al., 2012). It has also been shown that GOLGA8C replication on the $15 q 11.2$ region is associated with male infertility (Tu et al., 2013). The CNVs of patient Nos. 5190 and 3849, both with gains on 15q11.2, may result in an increase in replication leading to spermatogenic failure.

Huang et al. (2012) reported that the gene for DNA methyltransferase 3-like protein (DNMT3L) on 22q22.3 is essential for normal spermatogenesis and may be involved in spermatogenetic impairment and male infertility. They investigated allele, genotype, and haplotype frequencies of three SNP loci of DNMT3L to explore the possible association between DNMT3L and male infertility in 233 azoospermic males and 249 fertile controls. The results indicated that SNP rs2070565, as well as haplotypes AAA and GAA, may be associated with male infertility and that DNMT3L may contribute to azoospermia susceptibility in humans (Huang et al., 2012). In this study, two cases, Nos. 8235 and 8557, showed that the gain and loss of CNVs may lead to structural or functional changes of $D N M T 3 L$, resulting in azoospermia or oligozoospermia.

$A L F$ on $2 \mathrm{p} 16.3$ is highly expressed in adult testis and is located in the spermatid nuclei and in the annulus of spermatozoa. Huang et al. (2006) identified ALF as a human spermatogenesisrelated gene, and reported that the abnormal expression of $A L F$ might be a partial cause of human infertility. The $180 \mathrm{k}$ gain in CNVs observed in patient No. 5118 may affect normal expression of $A L F$, leading to azoospermia.

The CNVs involved in NPHP1 occurred on 2q13. It has previously been shown that defects in NPHP1 can lead to juvenile nephronophthisis type I, the most common genetic disorder causing end-stage renal failure in children and young adults (Hildebrandt et al., 1997). However, homozygous NPHP1 mutant mice were viable with renal manifestations of nephronophthisis and also appeared normal except for OT in infertile males. These novel findings indicate that nephrocystin is critical for the differentiation of early elongating spermatids into spermatozoa.

The azoospermic patient No. 7516 had a CNV gain of $540 \mathrm{k}$ on 8p12. Oral et al. (2008) identified NRG1 (neuregulin 1) as a novel FSH-upregulated clone homologous to mouse. NRG1 is known to control cell proliferation, differentiation, and survival in various tissues. Zhang et al. (2011) reported that $N R G 1$ promotes spermatogonial proliferation and initiates meiosis. It is assumed that the CNV on 8p12 involving NRG1 does not display normal function during spermiogenesis.

On the 12q12 locus, there are three genes expressed in the testis: AT-Rich Interaction Domain-Containing Protein 2 (ARID2), A Disintegrin-Like and Metalloproteinase with Thrombospondin Type 1 Motif 20 (ADAMTS20), and Twinfilin Drosophila Homolog of 1 (TWF1) (Ghorbel et al., 2013). The CNVs of patient No. 7516 may lead to certain changes in genetic structure or function, which may affect gene expression in testicular tissues and lead to spermatogenesis impairment.

Three spermatogenic failure-related genes on $17 \mathrm{p} 12$ of patient No. 7523 were COX10, MAK, and DNEL1. COX10 is expressed in multiple tissues with the highest expression occurring in the heart, skeletal muscle, and testis (Murakami et al., 1997). In situ hybridization confirmed that the mRNA expression of COX10 was stronger in the spermatogenic cells of normal fertile testes than azoospermic testes. It was suggested that COX10 may play a specific role in the development and progression of azoospermia (Yang et al., 2009). Expression of MAK was highly tissue-specific, and its transcripts were detected almost exclusively in testicular cells at the beginning and end of meiosis but hardly detectable in ovarian cells, including oocytes, after the dictyotene stage. These results suggest that MAK plays an important role in spermatogenesis (Matsushime et al., 1990). $D N E L 1$ is also expressed specifically in the testis, as determined by northern blot analysis of mRNA from 16 different tissues. The close similarity of DNEL1 to the C-terminal part of the axonemal beta- 
heavy chain may suggest an origin from a common progenitor gene, and the testis-specific pattern of expression indicates a possible role in sperm development and motility (Milisav et al., 1996). Further study is needed to determine if changes in expression of the above-mentioned genes result in azoospermia.

None of the described CNVs associated with spermatogenic failure in patients with chromosomal abnormalities and unexplained azoospermia were identified through high throughput genome-wide sequencing. In our present research, we provide evidence that CNVs contribute to the complex origin of male infertility and present a number of candidate genes that are potential risk factors for spermatogenic failure including EDDM3A and EDDM3B on 14q11.2, HLA-DRB1 and HLA-DQA1 on 6p21.32, POTE $B$ and GOLGA8C on 15q11.2, DNMT3L on 21q22.3, ALF on 2p16.3, NPHP1 on 2q13, NRG1 on 8p12, RID2, ADAMTS20 and TWF1 on 12q12, COX10, $M A K$, and DNEL1 on 17p12. However, further studies with larger sample sizes and different ethnic populations are necessary to confirm these findings, and further functional analyses are needed to elucidate the roles of these genes in pathological male infertility.

\section{ACKNOWLEDGMENTS}

We are grateful to the patients for participating in this study. We thank the staff of the Andrology, Cytogenetic and Molecular Laboratories for their excellent work. We also thank Berry Genomics, Beijing, China for their high throughput sequencing processing to determine CNVs. Research supported by funds from the National Natural Science Foundation of China (\#81471515).

\section{REFERENCES}

Carrell DT, De Jonge C and Lamb DJ (2006). The genetics of male infertility: A field of study whose time is now. Arch. Androl. 52: $269-274$.

Damyanova V, Dimitrova-Dikanarova D, Hadjidekova S, Savov A, et al. (2013). Genomic study in patients with idiopathic azoospermia and oligoasthenoteratozoospermia. Akush. Ginekol. 52: 27-34.

Fanciulli M, Petretto E and Aitman TJ (2010). Gene copy number variation and common human disease. Clin. Genet. 77 : 201-213.

Ferlin A, Raicu F, Gatta V, Zuccarello D, et al. (2007). Male infertility: Role of genetic background. Reprod. Biomed. Online 14: 734-745.

Feuk L, Carson AR and Scherer SW (2006). Structural variation in the human genome. Nat. Rev. Genet. 7: 85-97.

Freeman JL, Perry GH, Feuk L, Redon R, et al. (2006). Copy number variation: New insights in genome diversity. Genome. Res. 16: 949-961.

Ghorbel M, Baklouti-Gargouri S, EIGhazel H, Zribi N, et al. (2013). Pericentric inversion of chromosom 12 [Inv (12) (p12q12)] associated with idiopathic azoospermia in one infertile Tunisian man. Biochem. Biophys. Res. Commun. 432: 472-474.

Guediche N, Tosca L, Kara TA, Bas C, et al. (2012). Array comparative genomic hybridization analysis of small supernumerary marker chromosomes in human infertility. Reprod. Biomed. Online 24: 72-82.

Hildebrandt F, Otto E, Rensing C, Nothwang HG, et al. (1997). A novel gene encoding an SH3 domain protein is mutated in nephronophthisis type 1. Nat. Genet. 17: 149-153.

Hu Z, Li Z, Yu J, Tong C, et al. (2014). Association analysis identifies new risk loci for non-obstructive azoospermia in Chinese men. Nat. Commun. 5: 3857.

Huang JX, Scott MB, Pu XY and Zhou-Cun A (2012). Association between single-nucleotide polymorphisms of DNMT3L and infertility with azoospermia in Chinese men. Reprod. Biomed. Online 24: 66-71.

Huang M, Wang H, Li J, Zhou Z, et al. (2006). Involvement of ALF in human spermatogenesis and male infertility. Int. J. Mol. Med. 17: 599-604.

Ise T, Das S, Nagata S, Maeda H, et al. (2008). Expression of POTE protein in human testis detected by novel monoclonal antibodies. Biochem. Biophys. Res. Commun. 365: 603-608.

Jiang ST, Chiou YY, Wang E, Lin HK, et al. (2008). Targeted disruption of Nphp1 causes male infertility due to defects in the 
later steps of sperm morphogenesis in mice. Hum. Mol. Genet. 17: 3368-3379.

Jinam TA, Nakaoka H, Hosomichi K, Mitsunaga S, et al. (2013). HLA-DPB1*04:01 allele is associated with non-obstructive azoospermia in Japanese patients. Hum. Genet. 132: 1405-1411.

Kurpisz M, Nakonechnyy A, Niepieklo-Miniewska W, Havrylyuk A, et al. (2011). Weak association of anti-sperm antibodies and strong association of familial cryptorchidism/infertility with HLA-DRB1 polymorphisms in prepubertal Ukrainian boys. Reprod. Biol. Endocrinol. 9: 129.

Lee C, lafrate AJ and Brothman AR (2007). Copy number variations and clinical cytogenetic diagnosis of constitutional disorders. Nat. Genet. 39: S48-S54.

Martin RH (2008). Cytogenetic determinants of male fertility. Hum. Reprod. Update 14: 379-390.

Massart A, Lissens W, Tournaye H and Stouffs K (2012). Genetic causes of spermatogenic failure. Asian J. Androl. $14: 40-48$.

Matsushime H, Jinno A, Takagi N and Shibuya M (1990). A novel mammalian protein kinase gene (mak) is highly expressed in testicular germ cells at and after meiosis. Mol. Cell Biol. 10: 2261-2268.

McLachlan RI and O'Bryan MK (2010). Clinical Review\#: State of the art for genetic testing of infertile men. J. Clin. Endocrinol. Metab. 95: 1013-1024.

Milisav I, Jones MH and Affara NA (1996). Characterization of a novel human dynein-related gene that is specifically expressed in testis. Mamm. Genome 7: 667-672.

Murakami T, Reiter LT and Lupski JR (1997). Genomic structure and expression of the human heme a:farnesyltransferase (COX10) gene. Genomics 42: 161-164.

Oral O, Uchida I, Eto K, Nakayama Y, et al. (2008). Promotion of spermatogonial proliferation by neuregulin 1 in newt (Cynops pyrrhogaster) testis. Mech. Dev. 125: 906-917.

Redon R, Ishikawa S, Fitch KR, Feuk L, et al. (2006). Global variation in copy number in the human genome. Nature 444: 444-454.

Tu XD, Cong XW, Zeng J, Zheng DZ, et al. (2013). Analysis of small supernumerary marker chromosome $15 q 11$ in four infertile males. Zhonghua. Yi. Xue. Yi. Chuan. Xue. Za. Zhi. 30: 539-543.

Tuttelmann F, Simoni M, Kliesch S, Ledig S, et al. (2011). Copy number variants in patients with severe oligozoospermia and Sertoli-cell-only syndrome. PLoS One 6: e19426.

Van Assche E, Bonduelle M, Tournaye H, Joris H, et al. (1996). Cytogenetics of infertile men. Hum. Reprod. 11 (Suppl 4): 1-24, 25-26.

van der Ven K, Fimmers R, Engels G, van der Ven H, et al. (2000). Evidence for major histocompatibility complex-mediated effects on spermatogenesis in humans. Hum. Reprod. 15: 189-196.

World Health Organization (World Health Organization) (2010). WHO laboratory manual for the examination and processing of human semen. 5th ed. WHO, Geneva.

Yang B, Yuan JL, Gao XK, Wang H, et al. (2009). Expression of COX10 in human non-obstructive azoospermia testes. Zhonghua. Nan. Ke. Xue. 15: 599-603.

Zhang J, Eto K, Honmyou A, Nakao K, et al. (2011). Neuregulins are essential for spermatogonial proliferation and meiotic initiation in neonatal mouse testis. Development 138: 3159-3168.

Zhao H, Xu J, Zhang H, Sun J, et al. (2012). A genome-wide association study reveals that variants within the HLA region are associated with risk for nonobstructive azoospermia. Am. J. Hum. Genet. 90: 900-906. 